\title{
Primordial extremal black holes as dark matter
}

\author{
Yang Bai and Nicholas Orlofsky 1 \\ Department of Physics, University of Wisconsin-Madison, Madison, Wisconsin 53706, USA
}

(Received 14 August 2019; revised manuscript received 18 October 2019; accepted 18 February 2020; published 4 March 2020)

\begin{abstract}
We show that primordial (nearly) extremal black holes with a wide range of masses from the Planck scale to around $10^{9} \mathrm{~g}$ could be cosmologically stable and provide a viable explanation for dark matter, given a dark electromagnetism and a heavy dark electron. Hawking radiation and Schwinger discharge processes are suppressed by near extremality and the heaviness of the dark electron, respectively. The merger events of binary systems with opposite charges generate nonextremal black holes, whose subsequent Hawking evaporation produces transient neutrino and gamma ray signals to be observed at telescopes like IceCube and HAWC. The relationship between the near-extremal black hole and dark electron masses could also shed light on the weak gravity conjecture.
\end{abstract}

DOI: 10.1103/PhysRevD.101.055006

\section{INTRODUCTION}

Primordial black holes (PBHs) [1] are a compelling and simple explanation for dark matter (DM) [2-4]. They can take on a wide variety of masses, although several experiments constrain the allowable mass range [3,5,6], and there are proposals for future experiments to search much of the remaining parameter space $[7,8]$. Perhaps the most important constraint is that they survive today. Because BHs can evaporate via Hawking radiation [9], any Schwarzschild PBH with mass less than around $10^{15} \mathrm{~g}$ has a lifetime less than the age of the universe and cannot account for DM. A possible exception is that evaporation may cease when a $\mathrm{BH}$ reaches the Planck mass, leaving behind a Planck-scale relic [10] that could explain DM [11]. This is related to the information paradox [12], and its status is still under debate [13]. Even so, it would seem that PBHs cannot explain DM if they have mass between the Planck scale and $10^{15} \mathrm{~g}$, a mass range we will call "light."

To stabilize such a light PBH against Hawking radiation, one could consider a Reissner-Nordström (RN) $\mathrm{BH}$ charged under an unbroken $U(1)$ gauge symmetry. When the magnitude of the $U(1)$ charge $|Q|$ times the gauge coupling is equal to the $\mathrm{BH}$ mass in units of the Planck mass, the $\mathrm{BH}$ temperature is zero and the Hawking evaporation does not occur. Such a BH is

Published by the American Physical Society under the terms of the Creative Commons Attribution 4.0 International license. Further distribution of this work must maintain attribution to the author(s) and the published article's title, journal citation, and DOI. Funded by SCOAP ${ }^{3}$. called extremal ${ }^{1}$ (or eBH) and can serve as a DM candidate.

Accordingly, it may seem as if giving a PBH some large Standard Model (SM) electric charge could allow it to survive today with mass higher than the Planck scale. However, this is spoiled by the Schwinger effect [15], which allows pair production of electron-positron pairs in the strong electric field outside of the $\mathrm{eBH}$, leading to the eBH's discharge and subsequent evaporation [16-19]. In this paper, to evade Schwinger discharge effects, we explore the possibility of having a stable primordial (nearly) extremal black hole (PeBH) charged under an unbroken "dark quantum electrodynamics" (dQED) $U(1)_{\text {dark }}$ gauge symmetry, with its lightest charged state, or the "dark electron," much heavier than the SM electron.

\section{DARK QED AND EXTREMAL RN BLACK HOLES}

The Schwinger pair production rate per unit volume of dark electrons with mass $m_{e^{\prime}}$ and dark gauge coupling $e^{\prime}$ is $[15,19,20]^{2}$

\footnotetext{
${ }^{1}$ More generally, a Kerr-Newman BH can become extremal by a combination of its charge and angular momentum, while a Kerr BH is uncharged and can become extremal by its angular momentum alone. We do not consider these cases here because a nonextremal Kerr $\mathrm{BH}$ can lose angular momentum during its evaporation and will not approach extremal [14].

${ }^{2}$ This result is for a constant electric field. In later equations, we use the result including the radial dependence and curved metric derived in Refs. [19,20], which brings an extra $\mathcal{O}(1)$ factor in the logarithm of (2) and (3). See Refs. [21,22] for further discussion of Schwinger pair production in the presence of strong gravitational fields. Because these corrections are logarithmic, they do not affect our conclusions much.
} 


$$
\frac{d \Gamma_{\text {Schwinger }}}{d V}=\frac{\left(e^{\prime} E^{\prime}\right)^{2}}{4 \pi^{3}} \sum_{n=1}^{\infty} \frac{1}{n^{2}} \exp \left(-\frac{\pi n m_{e^{\prime}}^{2}}{e^{\prime} E^{\prime}}\right),
$$

with $E^{\prime}$ as the dark electric field. For a $\mathrm{RN} e \mathrm{eBH}$, which has dark charge $Q$ and mass $M_{\mathrm{eBH}}$ related by $e^{\prime} Q=\sqrt{4 \pi} M_{\mathrm{eBH}} / M_{\mathrm{pl}}$ with the Planck mass $M_{\mathrm{pl}}=$ $1 / \sqrt{G_{N}}=1.22 \times 10^{19} \mathrm{GeV}$ and $G_{N}$ Newton's constant, the outer horizon radius is $r_{+}=\left(M_{\mathrm{eBH}}+\right.$ $\left.\sqrt{M_{\mathrm{eBH}}^{2}-e^{\prime 2} Q^{2} M_{\mathrm{pl}}^{2} / 4 \pi}\right) / M_{\mathrm{pl}}^{2}=M_{\mathrm{eBH}} / M_{\mathrm{pl}}^{2}$. The corresponding dark electric field just outside the horizon is $E^{\prime}=M_{\mathrm{pl}}^{3} /\left(\sqrt{4 \pi} M_{\mathrm{eBH}}\right)$. Note that $E^{\prime}$ increases as $M_{\mathrm{eBH}}$ decreases, so the discharge speeds up as the PeBH discharges and evaporates. Requiring an evaporation time longer than the age of the Universe, $t_{\text {univ }} \approx 4.35 \times 10^{17} \mathrm{~s}$, one has $[19,20]$

$$
M_{\mathrm{eBH}}>M_{\mathrm{eBH}}^{\min } \approx \frac{e^{\prime} M_{\mathrm{pl}}^{3}}{2 \pi^{3 / 2} m_{e^{\prime}}^{2}} \ln \left(\frac{e^{\prime 3} M_{\mathrm{pl}} t_{\mathrm{univ}}}{32 \pi^{7 / 2}}\right) .
$$

For $\quad e^{\prime}=e=\sqrt{4 \pi \alpha}, \quad M_{\mathrm{eBH}}^{\min } \approx 6.3 \times 10^{25} \mathrm{GeV} \times$ $\left(10^{16} \mathrm{GeV} / m_{e^{\prime}}\right)^{2}$. Thus, for a heavy dark electron mass or a small gauge coupling, the PeBH can be very light. Note that if we apply the formula in (2) to SM QED with the electron mass $m_{e}=0.511 \mathrm{MeV}$, then $M_{\mathrm{eBH}}^{\min } \approx 10^{8} M_{\odot}$, which is already cosmologically stable without an extremal charge.

Naively, one can drive down the Schwinger discharge by arbitrarily decreasing $e^{\prime}$. On the other hand, this is constrained by the so-called "weak gravity conjecture" (WGC) [23,24], which suggests that $e^{\prime}>\sqrt{4 \pi} m_{e^{\prime}} / M_{\mathrm{pl}}$ for a particle with unit charge. Together with the WGC, the constraint in (2) becomes

$$
M_{\mathrm{eBH}}>M_{\mathrm{eBH}}^{\min , \mathrm{WGC}} \approx \frac{M_{\mathrm{pl}}^{2}}{\pi m_{e^{\prime}}} \ln \left(\frac{m_{e^{\prime}}^{3} t_{\mathrm{univ}}}{4 \pi^{2} M_{\mathrm{pl}}^{2}}\right) .
$$

Numerically, $M_{\mathrm{eBH}}^{\min , \mathrm{WGC}} \approx 5.5 \times 10^{23} \mathrm{GeV} \times\left(10^{16} \mathrm{GeV} / m_{e^{\prime}}\right)$. So, in principle, we could experimentally measure both $M_{\mathrm{eBH}}$ and $m_{e^{\prime}}$ to test the WGC.

While an exactly extremal $\mathrm{BH}$ has zero Hawking temperature, a nonextremal $\mathrm{BH}$ would take an infinite time to reach an extremal state. When the initial $\mathrm{BH}$ mass $M_{\mathrm{BH}}^{\text {init }} \gg M_{\mathrm{eBH}}$, the $\mathrm{BH}$ takes a time of $\tau_{\mathrm{BH}} \approx$ $(1 \mathrm{~s})\left(M_{\mathrm{BH}}^{\text {init }} / 10^{9} \mathrm{~g}\right)^{3}$ to evaporate towards very nearly extremal. After that, it asymptotically approaches extremal with time $t$-dependent Hawking temperature given by (see the Appendix A)

$$
T_{\mathrm{eBH}}(t) \approx \sqrt{\frac{60 M_{\mathrm{eBH}}}{\pi g_{*} t}} .
$$

Using the number of radiation degrees of freedom $g_{*} \sim 10$ and $t=t_{\text {univ }}$, the temperature is $T_{\mathrm{eBH}} \approx 1.3 \mathrm{eV} \times \sqrt{M_{\mathrm{eBH}} / \mathrm{g}}$. Generically, the evaporation from $M_{\mathrm{BH}}^{\text {init }}$ to nearly extremal emits more Hawking radiation over a short period of time, while the emission of the near-extremal BH is suppressed but longer in duration. As we will see, both these evaporation stages may provide observing opportunities.

\section{SEARCHING FOR ISOLATED PeBHs}

Assuming that PeBH's contribute a majority of the DM energy density, one could directly search for a $\mathrm{PeBH}$ in a laboratory. Using the whole Earth as a detector, the encounter rate of PeBH's is $\rho_{\mathrm{DM}} v_{\mathrm{DM}} \pi R_{\oplus}^{2} / M_{\mathrm{eBH}} \sim$ $1 /$ year $\times\left(10^{9} \mathrm{~g} / M_{\mathrm{eBH}}\right)$ with $\rho_{\mathrm{DM}} \approx 0.4 \mathrm{GeV} / \mathrm{cm}^{3}$ and $v_{\mathrm{DM}} \approx 10^{-3} c$. The geometric size of a light $\mathrm{PeBH}$ is very small such that its interaction length for scattering off nucleons in the Earth is very long: $2 \times 10^{11} \mathrm{~m} \times$ $\left(10^{9} \mathrm{~g} / M_{\mathrm{eBH}}\right)^{2}$. So, traditional DM direct detection methods like measuring nuclear recoil energy are not sensitive to PeBHs. Instead, it may eventually be possible to measure the gravitational effects of a $\mathrm{PeBH}$ passing by laser interferometers, mechanical resonators, or free-falling masses [25-27].

Rather than direct detection, near-extremal BHs could appear as pointlike astrophysical sources via their Hawking radiation. However, due to the small horizon radius for a light $\mathrm{PeBH}$, the electromagnetic radiation power has $\sim 2 \times 10^{-10} \mathrm{~W} \times\left(M_{\mathrm{eBH}} / 10^{9} \mathrm{~g}\right)^{4}$, which is too weak to be observed as a single source. PeBHs can also contribute to the diffuse photon flux. As detailed in Appendix B, present limits constrain $M_{\mathrm{eBH}} \lesssim 10^{12} \mathrm{~g}$ assuming they are most or all of DM.

Big bang nucleosynthesis (BBN) and the cosmic microwave background (CMB) can also constrain both stages of $\mathrm{PeBH}$ evaporation during the early Universe. For the generic case with $M_{\mathrm{BH}}^{\text {init }} \gg M_{\mathrm{eBH}}$, the evaporation of PBHs towards PeBHs may generate enough radiation energy to affect $\mathrm{BBN}$ or $\mathrm{CMB}$ observables. This is true for $M_{\mathrm{BH}}^{\text {init }} \gtrsim 1.4 \times 10^{9} \mathrm{~g}$, or equivalently $\tau_{\mathrm{BH}} \gtrsim 1 \mathrm{~s}$ when BBN starts. Because the PeBH is lighter than its initial $\mathrm{BH}$ mass, the constraints on the fraction of PeBHs making up DM today, $f_{\mathrm{eBH}}$, should be more stringent by a factor of $M_{\mathrm{BH}}^{\mathrm{init}} / M_{\mathrm{eBH}}$ compared to the existing constraints [5] on ordinary PBHs. On the other hand, for $M_{\mathrm{BH}}^{\text {init }} \lesssim 1.4 \times 10^{9}$ g, the initial $\mathrm{BH}$ has already reached the near-extremal state before $\mathrm{BBN}$, and there is no bound from the initial evaporation.

Emissions by $\mathrm{BHs}$ that have already reached near extremal are also constrained, albeit more weakly, owing to their reduced temperature. For example, if the Hawking temperature is high enough to produce hadrons, it will affect the light-element abundances. Rather than redo the detailed analysis, we make use of previous results to estimate the BBN bounds in the following way. For 
ordinary PBHs, the effects of hadron injection depend on the hadron emission rate $\mathrm{B}_{\mathrm{h}} n_{\mathrm{BH}}\langle E(t)\rangle^{-1} d M_{\mathrm{BH}} / d t$ with $\mathrm{B}_{\mathrm{h}}=\mathcal{O}(1)$ as the hadronic branching ratio and $\langle E(t)\rangle \sim$ $T_{\mathrm{BH}}(t)$ [28]. The most stringent constraint comes from the lithium-6/lithium-7 abundance ratio with $f_{\mathrm{BH}} \lesssim 6.8 \times 10^{-4}$ for $M_{\mathrm{BH}} \approx 4 \times 10^{10} \mathrm{~g}$ or $\tau_{\mathrm{BH}} \approx 2.6 \times 10^{4} \mathrm{~s}$, which we use as a reference point to derive the constraints on PeBHs. The constraint on $f_{\mathrm{eBH}}$ can be derived as $f_{\mathrm{eBH}} \lesssim 4.2 \times 10^{-3} \times$ $\left[\left(4 \times 10^{10} \mathrm{~g}\right) / M_{\mathrm{eBH}}\right]^{5 / 2}$. In practice, this constraint is generally superseded by constraints on the earlier evaporation from $M_{\mathrm{BH}}^{\text {init }}$.

Scaling the constraints for ordinary PBHs [5], we show the constraints on the present fraction of PeBHs as DM in Fig. 1 for several values of $r \equiv M_{\mathrm{BH}}^{\mathrm{init}} / M_{\mathrm{eBH}}$. The jumps in the exclusion curves relate to different element abundance ratios; the largest jump is where the $\mathrm{CMB}$ constraints become important. The constraint from the Hawking emission of the near-extremal BHs (as opposed to the Hawking radiation from the initial $\mathrm{BHs}$ ) is only important in Fig. 1 in the leftmost portion of the $r=3$ curve. One can see from Fig. 1 that there is a wide range of open parameter space (from the Planck scale to $\sim 10^{9} \mathrm{~g}$ ) to have PeBHs account for $100 \%$ of DM. We also show in the gray dotdashed lines the combined theoretical constraints from Schwinger evaporation together with the WGC from Eq. (3). If the WGC is not valid, one can relax the Schwinger-evaporation constraints by choosing a tiny gauge coupling $e^{\prime}$ [see Eq. (2)]. If both $m_{e^{\prime}}$ and $M_{\mathrm{eBH}}$ can be measured, one could test the WGC experimentally. While

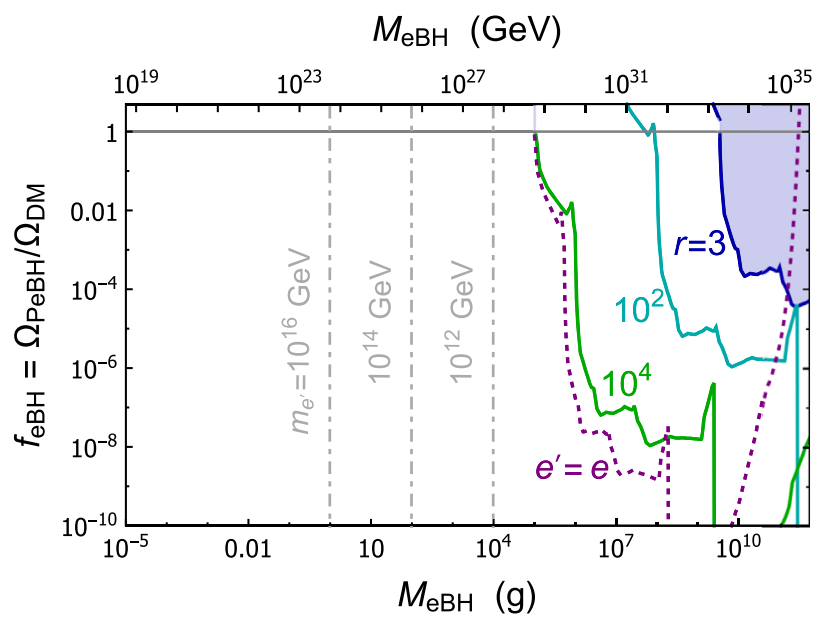

FIG. 1. The BBN and other BH evaporation-related constraints on the present $\mathrm{PeBH}$ over dark matter energy density ratio $f_{\mathrm{eBH}}$ as a function of $\mathrm{PeBH}$ masses for various ratios of the initial $\mathrm{BH}$ mass over the PeBH mass $r \equiv M_{\mathrm{BH}}^{\mathrm{init}} / M_{\mathrm{eBH}}$. The purple dotted line shows the constraints for one possible $\mathrm{PeBH}$ cosmological formation scenario described around (9) with $e^{\prime}=e$. In the gray dot-dashed lines, potential theoretical constraints from Schwinger evaporation and the WGC via (3) are shown for different dark electron masses. not displayed on the plot, when $r \rightarrow 1$ (meaning the $\mathrm{BH}$ was born very near extremal), the BBN constraints are dramatically relaxed by a factor of $\left[\left(M_{\mathrm{BH}}^{\mathrm{init}}-M_{\mathrm{eBH}}\right) / M_{\mathrm{eBH}}\right]^{3 / 2}$. $^{3}$

\section{SIGNATURES OF BINARY PeBH MERGERS}

Other than signatures from isolated PeBHs, one could also search for signatures from binary PeBH systems. Although the merger of a light PeBH binary generates gravitational waves, the signal flux is too small to be detected. On the other hand, the PeBH binary system typically contains both positively and negatively charged PeBHs (like-charged PeBHs have no net force to bind them). When they merge, the charges are neutralized such that the final $\mathrm{BH}$ becomes nonextremal and can provide striking Hawking radiation signatures.

Given the large uncertainties on estimating the $\mathrm{BH}$ binary merging rate [29], which are most severe when $f_{\mathrm{eBH}}=\mathcal{O}(1)$ and come predominantly from binary disruption both immediately after binary formation and during halo formation, we simply make a phenomenological parametrization for the rate and discuss some benchmark-model estimation at the end of this section. For an approximately monochromatic $\mathrm{PeBH}$ mass distribution with mass $M_{\mathrm{eBH}}$, the averaged binary merging rate can be parametrized as

$$
\Gamma_{\text {merge }}=\Gamma_{0} f_{\mathrm{eBH}}^{a}\left(\frac{M_{\mathrm{eBH}}}{M_{\mathrm{ref}}}\right)^{b}\left(\frac{t}{t_{\text {univ }}}\right)^{c},
$$

where the exponents $a, b, c$ depend on the binary formation history. Ignoring the disruption effects in the galaxy formation, the merging rate per volume in the current Milky Way galaxy is

$$
R(r)=\frac{\rho_{\mathrm{DM}}(r)}{4 M_{\mathrm{eBH}}} \Gamma_{0} f_{\mathrm{eBH}}^{a+1}\left(\frac{M_{\mathrm{eBH}}}{M_{\mathrm{ref}}}\right)^{b},
$$

with one factor of $1 / 2$ to avoid overcounting, and the second factor of $1 / 2$ because only oppositely charged PeBHs form binaries.

After two $+Q$ and $-Q$ PeBH's merge, and ignoring the angular momentum, they become approximately an ordinary $\mathrm{BH}$ with a mass of $M_{\mathrm{BH}} \approx 2 M_{\mathrm{eBH}}$. As the $\mathrm{BH}$ evaporates, the Hawking temperature, $T_{\mathrm{BH}} \approx 53 \mathrm{TeV} \times$ $\left(10^{8} \mathrm{~g} / M_{\mathrm{eBH}}\right)$, is much higher than that of an isolated $\mathrm{PeBH}$. The duration of the evaporation is very short: $\tau_{\mathrm{BH}} \approx 10^{-3} \mathrm{~s} \times\left(M_{\mathrm{eBH}} / 10^{8} \mathrm{~g}\right)^{3}$. So, the final BH evaporation is a very transient event.

Both high-energy neutrinos and gamma rays are good signatures to search for the final evaporation of the merged

\footnotetext{
${ }^{3}$ We regard the possibility that the PBHs are born in an already near-extremal state as less likely based on the formation mechanism presented later. However, if a formation model allowing $r$ near unity is realized, its evaporation would be less constraining.
} 
binary system. Under several assumptions detailed in Appendix B, the neutrino flux is estimated as $\Phi_{\nu} \approx$ $5 \times 10^{-15} \mathrm{~cm}^{-2} \mathrm{sr}^{-1} \mathrm{~s}^{-1}\left(M_{\mathrm{eBH}} / 10^{8} \mathrm{~g}\right)^{1+b}\left(f_{\mathrm{eBH}}^{a+1} \Gamma_{0} / 10^{-30} \mathrm{~s}^{-1}\right)$.

The lack of an observation of neutrinos above the $\mathrm{PeV}$ scale by IceCube [30] along with constraints from ANITA [31] and NuMoon [32,33] limit $\Phi_{\nu} \lesssim(2.7 \times$ $\left.10^{-15} \mathrm{~cm}^{-2} \mathrm{~s}^{-1} \mathrm{sr}^{-1}\right)\left(E_{\nu} / 10^{7} \mathrm{GeV}\right)^{-4 / 5}$ for $10^{7} \mathrm{GeV} \lesssim$ $E_{\nu} \lesssim 10^{16} \mathrm{GeV}$. Further, the observation of cosmic neutrinos by IceCube with energies $10^{4} \mathrm{GeV} \lesssim E_{\nu} \lesssim 10^{7} \mathrm{GeV}$ [34] can be used to set an upper limit $\Phi_{\nu} \lesssim$ $\left(5.9 \times 10^{-16} \mathrm{~cm}^{-2} \mathrm{~s}^{-1} \mathrm{sr}^{-1}\right)\left(E_{\nu} / 10^{7} \mathrm{GeV}\right)^{-8 / 5}$. This leads to a constraint on the merging rate and present $\mathrm{PeBH}$ abundance of roughly ${ }^{4}$

$\Gamma_{0} f_{\mathrm{eBH}}^{a+1} \lesssim \begin{cases}\left(10^{-29} \mathrm{~s}^{-1}\right)\left(\frac{10^{8} \mathrm{~g}}{M_{\mathrm{eBH}}}\right)^{\frac{1}{5}+b}, & M_{\mathrm{eBH}} \in\left(10^{-3}, 10^{6}\right) \mathrm{g} \\ \left(5 \times 10^{-29} \mathrm{~s}^{-1}\right)\left(\frac{10^{8} \mathrm{~g}}{M_{\mathrm{eBH}}}\right)^{b-\frac{3}{5}}, & M_{\mathrm{eBH}} \in\left(10^{6}, 10^{9}\right) \mathrm{g} .\end{cases}$

Comparing against Fig. 1, this bound extends down in mass to the lower limits on $M_{\mathrm{eBH}}$ coming from the WGC, and up in mass to PeBHs that are generally constrained by the evaporation from $M_{\mathrm{BH}}^{\text {init }}$. In principle, one could search for still higher-energy neutrinos to get down to $M_{\mathrm{eBH}} \sim M_{\mathrm{pl}}$, or use lower-energy gamma ray bounds from HAWC [36,37] and CTA [38] to extend to higher $M_{\mathrm{eBH}}$.

Other than searching for only high-energy neutrinos or only gamma rays, one could also use the multimessenger approach to search for coincident cosmic ray events by several telescopes: IceCube, ANITA, NuMoon, HAWC, CTA, Fermi-LAT [39,40], Pierre Auger [41], and others $[42,43]$. This would be a particularly powerful discovery channel that can discriminate against other high-energy backgrounds and allow us to directly detect Hawking radiation.

An estimate for the merger rate is derived in Appendix C, which has $a=1 / 3, b=1 / 9, c=-8 / 9$, and $\Gamma_{0} \approx 1.5 \times$ $10^{-23} \mathrm{~s}^{-1}$ for $M_{\text {ref }}=10^{8} \mathrm{~g}$ (with a monochromatic mass distribution) and numerical factors $\alpha=\beta=1$ as defined in Appendix C. This estimate assumes that the PeBHs in the to-be-formed binary decouple from Hubble expansion shortly before matter-radiation equality and form longlived binaries due to the tidal force of neighboring PeBHs. Naively, it seems to be very constrained by (7). However, as argued in Ref. [29], these binaries may be disrupted in the early Universe when $f_{\mathrm{eBH}}=\mathcal{O}(1)$, resulting in a lengthened merger time and a dramatically decreased merger rate-by their estimate, a decrease of up to but not larger

\footnotetext{
${ }^{4}$ As a cross-check, this bound is fairly similar to the lifetime constraint on decaying DM made by IceCube [35], though the latter included both halo and extragalactic components as well as a fuller accounting of all neutrino production channels.
}

than a factor of $10^{17}$ for $M_{\mathrm{eBH}}=10^{8}$ g. ${ }^{5}$ Further disruptions are likely during halo formation. Thus, we expect that our estimation for $\Gamma_{0}$ may severely overestimate the merger rate.

\section{PeBH FORMATION}

So far, we have remained agnostic to how the population of PeBHs is formed and focused instead on the phenomenology. Here, we highlight one possibility for their formation relying on the statistical distribution of charges in the early Universe.

Assume that the PBHs are initially produced during radiation domination following reheating. $\mathrm{PBHs}$ form as a result of primordial energy overdensities that collapse almost immediately after reentering the horizon. These overdensities could be seeded as the result of any number of already or as-yet proposed inflationary or postinflationary models including, e.g., running mass inflation [44-46], parametric resonance [47-49], or curvatons [50,51] (see $[3,4,52]$ for reviews). Then, the initial PBH mass is related to the scale (and thus temperature $T_{\text {form }}$ ) at which the overdensities reenter the horizon by [3]

$$
\begin{aligned}
M_{\mathrm{BH}}^{\mathrm{init}} & \approx \frac{4 \pi}{3} \frac{\gamma \rho_{r}}{H^{3}} \\
& \approx 10^{9} \mathrm{~g}\left(\frac{\gamma}{0.2}\right)\left(\frac{10^{11} \mathrm{GeV}}{T_{\text {form }}}\right)^{2}\left(\frac{g_{*}}{100}\right)^{-1 / 2},
\end{aligned}
$$

with $H=\left[(8 \pi / 3) \rho_{r} / M_{\mathrm{pl}}^{2}\right]^{1 / 2}$ the Hubble parameter, $\rho_{r}$ the radiation energy density, $g_{*}$ the effective radiation degrees of freedom, and $\gamma \approx 0.2$ a numerical factor depending on the details of the collapse [53]. Note that $T_{\text {form }}<T_{\mathrm{RH}} \lesssim$ $10^{16} \mathrm{GeV}$ (with $T_{\mathrm{RH}}$ the reheating temperature) is required by nonobservation of tensor perturbations in the CMB [54].

We further assume that the dark electrons are still relativistic when the PBHs form, having temperature $T_{\text {form }}^{\prime}>m_{e^{\prime}}$. Note that the dark sector temperature $T^{\prime}$ may not be equal to the visible sector temperature $T$. Since the dark photon is massless, the constraints on additional radiation degrees of freedom from CMB measurement require $T^{\prime} / T \lesssim 0.46$ [55].

One may then ask what the dark charge of these to-becollapsed regions is as they reenter. We take the ansatz that reheating occurs in such a way that the dark electrons and positrons each follow independent Poisson distributions in superhorizon volumes. Thus, the average number of each within a volume that is just reentering the horizon is $\left\langle N_{\text {col }}\right\rangle \approx \frac{4 \pi}{3} n_{e^{\prime}} H^{-3}$. Using (8), the average number of a

\footnotetext{
${ }^{5}$ Their analysis only considered gravitation. Besides accounting for the change in merger time, properly adding dQED would require a completely separate N-body simulation because samecharged PeBHs do not interact.
} 
particular species (either dark electron or positron) within the collapsed volume is

$$
\left\langle N_{\text {col }}\right\rangle \approx 3 \times 10^{20} \times\left(\frac{M_{\mathrm{BH}}^{\mathrm{init}}}{10^{9} \mathrm{~g}}\right)^{3 / 2}\left(\frac{T^{\prime}}{T}\right)^{3} .
$$

If the dark electron and positron distributions are indeed Poisson and independent, the resulting charge distribution of the PBHs is a Skellam distribution [56] with mean $\langle Q\rangle=0 \quad$ and standard deviation $\sigma_{Q, \mathrm{col}}=\sqrt{2\left\langle N_{\mathrm{col}}\right\rangle}$. Moreover, if there are multiple dark-charged fermions with $g_{i}$ relativistic degrees of freedom (at $T_{\text {form }}^{\prime}$ ) and charge $q_{i}$, then the typical charge is $Q \sim \sigma_{Q \text {,col }}=\sqrt{\left\langle N_{\text {col }}\right\rangle \sum_{i} g_{i} q_{i}}$. Additionally, if there is some correlation between the energy density perturbation and charge asymmetry fluctuation, the typical charge could scale as $Q \sim \eta\left\langle N_{\text {col }}\right\rangle$, with $\eta$ a model-dependent number to quantify the charge asymmetry.

After the formation of the initial nonextremal BHs, the ambient dark electrons and positrons can be further absorbed and change the charge distribution. Assuming that the PBH absorbs both charges in equal numbers, no discharge occurs. This can be arranged, e.g., if the dark electrons efficiently annihilate between when the PBH forms and when it evaporates to near extremal (see Appendix E for details). Then, the number of dark electrons and positrons absorbed is

$$
N_{\mathrm{abs}} \approx 4 \times 10^{18}\left(\frac{M_{\mathrm{BH}}^{\mathrm{init}}}{10^{9} \mathrm{~g}}\right)^{3 / 2}\left(\frac{T^{\prime}}{T}\right)^{3}\left(\frac{g_{*}}{100}\right)^{-3 / 4} .
$$

This results in a one-dimensional random walk for the charge accumulation with $N_{\text {abs }}$ steps, which is characterized by a standard deviation of $\sigma_{Q, \text { abs }}=\sqrt{N_{\text {abs }} \sum_{i}\left(g_{i} / 2\right) q_{i}}$. It is subdominant to the charge from the initial collapse in (9) and provides an additional small contribution to $Q$.

In the case without too much discharge, we may expect a typical PeBH mass of $M_{\mathrm{eBH}} \approx e^{\prime} Q M_{\mathrm{pl}}$ if it is above $M_{\mathrm{eBH}}^{\min }$ in Eq. (2); otherwise, if it is below the evaporation bound, the surviving $\mathrm{PeBH}$ masses will be peaked just slightly above $M_{\mathrm{eBH}}^{\min }$. Constraints assuming negligible discharge are shown in Fig. 1 for $e^{\prime}=e$. The PeBH abundance is directly related to the probability to form the initial $\mathrm{PBHs}$, which scales exponentially with the density perturbation amplitude $[4,52]$ and can account for part or all of DM.

\section{DISCUSSION AND CONCLUSIONS}

In the model considered here to stabilize light PeBHs against discharge, the dark electron is assumed to be the lightest dQED-charged particle and hence stable. However, if the very heavy dark electron has a thermal relic abundance set by its annihilation into dark photons, it will overclose the universe. This issue can be resolved by introducing additional non-Abelian low-scale confining gauge charges for the dark electrons (see, e.g., Refs. [57,58]).

So far, we have only considered the gravitational interaction between the visible and dark sectors. Additional interactions like kinetic mixing between the photon $(F)$ and dark photon $\left(F^{\prime}\right)$ of the form $\epsilon F_{\mu \nu} F^{\prime \mu \nu}$ [59] can make dark electrons and PeBHs millicharged under ordinary electromagnetism. However, in order for Schwinger pair production of SM electrons to be inefficient, the mixing parameter is required from Eq. (2) to be $\epsilon \lesssim 1.6 \times 10^{-19}$ (independent of the small $M_{\mathrm{eBH}}$ considered here). Such a small mixing parameter can be realized in certain models (see, e.g., Ref. [60]). So far, no experiments probe dark photons with this range of $\epsilon$ [61]. Because the PeBH's SM electromagnetic charge is $\epsilon \sqrt{4 \pi} M_{\mathrm{eBH}} /\left(e M_{\mathrm{pl}}\right)$, it is millicharged and difficult to detect experimentally.

In summary, we have studied the possibility of having PeBHs as a DM candidate based on a dQED model with a heavy dark electron. A wide range of $\mathrm{PeBH}$ masses from the Planck scale to around $10^{9} \mathrm{~g}$ are still allowed by the experimental constraints. The merger event of two equalmass PeBHs with opposite charges can generate a neutral and light Schwarzschild BH whose fast Hawking evaporation can provide transient signatures to be detected by various telescopes. While a simple early-universe formation story for PeBHs has been discussed in this paper, other formation mechanisms based on different histories are worthy of future studies.

\section{ACKNOWLEDGMENTS}

This work is supported by the U.S. Department of Energy under the Contract No. DE-SC0017647. Part of this work was performed at the Aspen Center for Physics, which is supported by National Science Foundation Grant No. PHY-1066293.

\section{APPENDIX A: PeBH TEMPERATURE}

The Hawking temperature for a $\mathrm{RN} \mathrm{BH}$ is

$T\left(M_{\mathrm{BH}}, M_{\mathrm{eBH}}\right)=\frac{M_{\mathrm{pl}}^{2}}{2 \pi} \frac{\sqrt{M_{\mathrm{BH}}^{2}-M_{\mathrm{eBH}}^{2}}}{\left(M_{\mathrm{BH}}+\sqrt{M_{\mathrm{BH}}^{2}-M_{\mathrm{eBH}}^{2}}\right)^{2}}$.

Using the blackbody radiation formula, the $\mathrm{BH}$ mass loss rate is (see Refs. [62,63] for spin- and charge-dependent corrections)

$$
\frac{d M_{\mathrm{BH}}}{d t} \approx-\frac{\pi^{2}}{120} g_{*} 4 \pi r_{+}^{2}\left[T\left(M_{\mathrm{BH}}, M_{\mathrm{eBH}}\right)\right]^{4},
$$

with $g_{*}$ the radiation degrees of freedom. When the initial $\mathrm{BH}$ mass $M_{\mathrm{BH}}^{\text {init }} \gg M_{\mathrm{eBH}}$ and ignoring the change of $g_{*}$, the $\mathrm{BH}$ will evaporate to very nearly extremal with a lifetime 
$\tau_{\mathrm{BH}} \approx 5120 \pi\left(M_{\mathrm{BH}}^{\mathrm{init}}\right)^{3} /\left(g_{*} M_{\mathrm{pl}}^{4}\right) \approx(1 \mathrm{~s})\left(M_{\mathrm{BH}}^{\mathrm{init}} / 10^{9} \mathrm{~g}\right)^{3}$. After that, the $\mathrm{BH}$ mass evolves with time as

$$
M_{\mathrm{BH}}(t) \approx M_{\mathrm{eBH}}+\frac{120 \pi M_{\mathrm{eBH}}^{4}}{g_{*} M_{\mathrm{pl}}^{4} t} .
$$

Using this in Eq. (A1) leads to the result in Eq. (4).

\section{APPENDIX B: COSMIC RAY FLUX CALCULATIONS}

First, consider the emission of photons by near-extremal PeBHs. For a Boltzmann energy spectrum with a temperature $T_{\mathrm{eBH}}$, the averaged energy of the radiated photons has $\left\langle E_{\gamma}\right\rangle \approx 2.7 T_{\mathrm{eBH}}$. The photon spectral emission rate from one $\mathrm{PeBH}$ has

$$
\frac{d N_{\gamma}}{d E_{\gamma} d t} \approx \frac{\pi^{2}}{60} 4 \pi r_{+}^{2} T_{\mathrm{eBH}}^{4} \frac{1}{\left\langle E_{\gamma}\right\rangle^{2}} \approx \frac{0.54 M_{\mathrm{eBH}}^{3}}{M_{\mathrm{pl}}^{4} t_{\mathrm{univ}}},
$$

which has a higher rate for a heavier BH mass.

Within our Galaxy and similar to a decaying DM model, one can adopt the normalized " $J$ "-factor after integrating along the line of sight to calculate the $\mathrm{PeBH}$ radiated flux

$$
\hat{J} \equiv \frac{1}{4 \pi M_{\mathrm{DM}}^{\text {total }}} \int \cos b \mathrm{~d} b \mathrm{~d} \ell \mathrm{d} s \rho_{\mathrm{DM}}[r(s, \ell, b)],
$$

with $M_{\mathrm{DM}}^{\text {total }} \equiv 4 \pi \int \mathrm{d} r r^{2} \rho_{\mathrm{DM}}(r)$. Here, $-\pi / 2 \leq b<\pi / 2$ and $0 \leq \ell<2 \pi$ are galactic latitude and longitudinal angles; $s$ is the line-of-sight distance with $r^{2}=s^{2} \cos ^{2} b+R_{\odot}^{2}-$ $2 s R_{\odot} \cos \ell \cos b$ and the distance of the Sun to the Galactic center $R_{\odot} \approx 8.5 \mathrm{kpc}$. Using the Einasto profile with $\beta=0.17$ [64], the solid-angle-averaged $J$-factor has $\hat{J} \approx 1 /(33 \mathrm{kpc})^{2} \mathrm{sr}^{-1}$. Combining with (B1), the contribution to the sky-averaged diffuse photon spectral flux is estimated by

$$
\begin{aligned}
\phi_{\gamma} & \approx \frac{M_{\mathrm{DM}}^{\text {total }}}{M_{\mathrm{eBH}}} \hat{J} \frac{d N_{\gamma}}{d E_{\gamma} d t} \\
& \approx 9 \times 10^{-11} \mathrm{~cm}^{-2} \mathrm{sr}^{-1} \mathrm{~s}^{-1} \mathrm{MeV}^{-1} \times\left(\frac{M_{\mathrm{eBH}}}{10^{9} \mathrm{~g}}\right)^{2},
\end{aligned}
$$

for the averaged photon energy of $\left\langle E_{\gamma}\right\rangle \approx 0.4 \mathrm{MeV} \times$ $\left[M_{\mathrm{eBH}} /\left(10^{9} \mathrm{~g}\right)\right]^{1 / 2}$. Comparing to the spectra of the isotropic diffuse gamma ray measured by the COMPTEL Collaboration [65] and the EGRET data [66,67], the PeBH mass is constrained to be $M_{\mathrm{eBH}} \lesssim 10^{12} \mathrm{~g}$ assuming that they compose all or nearly all of DM.

A similar calculation gives the high-energy neutrino (and gamma ray) flux from ordinary uncharged $\mathrm{BHs}$, which we use to estimate bounds on PeBH mergers. The number of neutrinos from one evaporation event is estimated to be $\eta_{\nu}\left(2 M_{\mathrm{eBH}}\right) /\left\langle E_{\nu}\right\rangle$ with the averaged neutrino energy $\left\langle E_{\nu}\right\rangle \approx 4.22 T_{\mathrm{BH}} \approx 220 \mathrm{TeV} \times\left(10^{8} \mathrm{~g} / M_{\mathrm{eBH}}\right)$ and $\eta_{\nu} \approx 0.06$ the fraction of energy into primary threeflavor neutrinos after taking into account the different thermally averaged cross sections [62]. Including the averaged binary merger rate, the neutrino emission rate is estimated to be

$$
\frac{d N_{\nu}}{d t} \approx 1.4 \frac{M_{\mathrm{eBH}}^{2}}{M_{\mathrm{pl}}^{2}} \Gamma_{\text {merge }}
$$

This only accounts for the primary neutrinos, ignoring secondary neutrinos produced in the cascade decays of other Hawking-emitted particles. Using the normalized $J$-factor of the Einasto profile, the predicted high-energy neutrino flux originating from the Milky Way halo is

$$
\begin{aligned}
\Phi_{\nu} & \approx \frac{M_{\mathrm{DM}}^{\mathrm{total}}}{4 M_{\mathrm{eBH}}} \hat{J} \frac{d N_{\nu}}{d t} \\
& \approx 5 \times 10^{-15} \mathrm{~cm}^{-2} \mathrm{sr}^{-1} \mathrm{~s}^{-1}\left(\frac{M_{\mathrm{eBH}}}{10^{8} \mathrm{~g}}\right)^{1+b}\left(\frac{f_{\mathrm{eBH}}^{a+1} \Gamma_{0}}{10^{-30} \mathrm{~s}^{-1}}\right) .
\end{aligned}
$$

The extragalactic flux may be comparable to or a couple of orders of magnitude smaller than the galactic flux-see, e.g., [68]. Note this treatment makes the important but unqualified assumption that the binary merger distribution identically follows the DM distribution. This is less likely to be true within DM halos, where disruptions during halo formation may suppress the merger rate in the Milky Way halo. Since the extragalactic binary population is more stable against these disruptions, their mergers may provide more robust constraints on PeBHs if the extragalactic flux is similar to or within a couple of orders of magnitude of the undisrupted galactic flux [68]. Further work is needed to understand the halo disruptions. On the other hand, a merger occurring nearby in the Milky Way halo or one of its dwarf galaxies may be more likely to be detected with several instruments observing different messengers. Thus, nearby mergers may offer better discovery prospects.

\section{APPENDIX C: BINARY MERGER RATE}

The PeBHs may attract each other via gravitational and dQED forces to form binaries in the early universe. Let us take the simplifying assumption that PeBHs form during radiation domination, and the first period of matter domination is the ordinary one occurring at redshift $z_{\text {eq }} \approx 3400$ [55]. In order to decouple from Hubble expansion, a pair of PeBHs must dominate their local energy density. Thus, such binaries only form when $z>z_{\mathrm{eq}}{ }^{6}$. They may subsequently merge, and their merger rate can be estimated.

\footnotetext{
${ }^{6}$ The merger rate of binaries formed in galactic halos is subdominant. For example, using the estimate in Ref. [69] for the purely gravitational case, the halo-formed binary merger rate is about 5 orders of magnitude smaller for $M_{\mathrm{eBH}}=10^{8} \mathrm{~g}$.
} 
To calculate the merger rate, we update the treatment in $[70,71]$ (although see further refinements in $[29,69]$ ) to account for radiation due to dQED, which dominates the gravitational radiation. First, the average separation at matter-radiation equality is

$$
\begin{aligned}
\bar{x} & =\left(\frac{M_{\mathrm{eBH}}}{\rho_{\mathrm{PeBH}}\left(z_{\mathrm{eq}}\right)}\right)^{1 / 3} \\
& =\frac{f_{\mathrm{eBH}}^{-1 / 3}}{\left(1+z_{\mathrm{eq}}\right)}\left(\frac{8 \pi M_{\mathrm{eBH}}}{3 M_{\mathrm{pl}}^{2} H_{0}^{2} \Omega_{\mathrm{DM}}}\right)^{1 / 3} .
\end{aligned}
$$

A pair of nearest-neighbor PeBHs form into a binary if $x<f_{\mathrm{eBH}}^{1 / 3} \bar{x}$ depending on the distance to the next-nearestneighbor PeBH $y$ (which provides a tidal force to prevent the other two PeBHs from immediately merging). The resulting orbital parameters are [70]

$a=\frac{\alpha}{f_{\mathrm{eBH}}} \frac{x^{4}}{\bar{x}^{3}}, \quad b=\beta\left(\frac{x}{y}\right)^{3} a, \quad e=\sqrt{1-\left(\frac{b}{a}\right)^{2}}$,

indicating the semimajor axis, semiminor axis, and eccentricity, respectively, with $\alpha$ and $\beta=\mathcal{O}(1)$ numbers that should be determined through numerical simulations. Then, the merger time for two PeBHs with masses $M_{\mathrm{eBH}}=$ $M_{1,2}$ is [see the detailed derivation in the next section leading to (D6)]

$$
\begin{aligned}
t & =\frac{1}{16} \frac{M_{\mathrm{pl}}^{4}}{M_{1} M_{2}} a^{3} \frac{\left(1-e^{2}\right)^{5 / 2}}{2+e^{2}} \equiv \bar{t} f_{\mathrm{eBH}}^{3}\left(\frac{a}{\bar{x}}\right)^{3} \frac{\left(1-e^{2}\right)^{5 / 2}}{\left(2+e^{2}\right) / 3} \\
& \approx \alpha^{3} \beta^{5} \bar{t}\left(\frac{x}{\bar{x}}\right)^{27}\left(\frac{y}{\bar{x}}\right)^{-15},
\end{aligned}
$$

where in the last line we approximate $e \approx 1$, which is valid for mergers occurring today. Here, the timescale $\bar{t} \equiv M_{\mathrm{pl}}^{4} \bar{x}^{3} /\left(48 f_{\mathrm{eBH}}^{3} M_{1} M_{2}\right)$. This can be compared to the merger time for ordinary, uncharged PBHs emitting only gravitational radiation for $e \approx 1$ [72],

$$
t_{\text {grav }}=\frac{3}{85} \frac{M_{\mathrm{pl}}^{6}}{M_{1} M_{2}\left(M_{1}+M_{2}\right)} a^{4}\left(1-e^{2}\right)^{7 / 2} .
$$

Taking $M_{1}=M_{2}=M$, their ratio is

$$
\begin{aligned}
\frac{t_{\text {grav }}}{t} & =\frac{72}{85} \frac{M_{\mathrm{pl}}^{2}}{M} a\left(1-e^{2}\right) \frac{2+e^{2}}{3} \\
& \approx 2 \times 10^{14}\left(\frac{10^{9} \mathrm{~g}}{M}\right)\left(\frac{a}{f_{\mathrm{eBH}}^{1 / 3} \bar{x}}\right)\left(\frac{1-e^{2}}{10^{-14}}\right) \frac{2+e^{2}}{3},
\end{aligned}
$$

where fiducial values of $a$ (with $\bar{x}$ evaluated using $M=10^{9} \mathrm{~g}$ ) and $1-e^{2}$ are chosen so that $t \approx t_{\text {univ }}$ (the full probability distribution for these is considered below).
As expected, the $\mathrm{dQED}$ radiation makes the binary merge more quickly than gravity alone.

The normalized probability distribution is given by [70]

$$
d P=\frac{9 x^{2} y^{2}}{\bar{x}^{6}} e^{-(y / \bar{x})^{3}} d x d y .
$$

After changing variables from $x$ to $t$ using (C3) and properly taking care of the integration region of $y$, we integrate $0<x<f_{\mathrm{eBH}}^{1 / 3} \bar{x}, x<y<\infty$ to arrive at the merging rate as

$$
\begin{aligned}
\Gamma_{\text {merge }} & =\frac{d P}{d t}=\frac{1}{9}\left(\frac{t}{\alpha^{3} \beta^{5} \bar{t}}\right)^{1 / 9} \frac{1}{t} \\
& \times\left\{\Gamma\left[\frac{14}{9},\left(\frac{t}{\alpha^{3} \beta^{5} \bar{t}}\right)^{1 / 4}\right]-\Gamma\left[\frac{14}{9},\left(\frac{t}{\alpha^{3} \beta^{5} \bar{t}}\right)^{-1 / 5} f_{\mathrm{eBH}}^{9 / 5}\right]\right\},
\end{aligned}
$$

with $\Gamma[i, z]$ the incomplete gamma function.

The merger rate per unit volume is then

$$
R=\frac{n_{\mathrm{PeBH}}}{4} \frac{d P}{d t}\left(t=t_{\mathrm{univ}}\right),
$$

where one factor of $1 / 2$ is to avoid overcounting, and the second factor is to account for the fact that only oppositely charged PeBHs form binaries. It is often sufficient to approximate the $\Gamma$ function as a constant, in which case

$$
R \propto f_{\mathrm{eBH}}^{13 / 9} M_{\mathrm{eBH}}^{-8 / 9} t^{-8 / 9} .
$$

Note that this equation matches the general structure of (7). By contrast, for uncharged PBHs, $R \propto f_{\mathrm{eBH}}^{53 / 37} M^{-32 / 37} t^{-32 / 37}$. While the exponents are numerically similar, the overall normalization has a merger rate for charged PeBHs about 2 orders of magnitude larger than that of ordinary PBHs for the lighter masses of interest here [note that the lifetime only enters as $\bar{t}^{-1 / 9}$ in $\Gamma_{\text {merge }}$ in (C7), and a similarly small exponent for the gravitational case, which explains the relationship to (C5)].

\section{APPENDIX D: dQED RADIATION}

To calculate the merger time for PeBH binaries, consider a central inverse-square force acting on a body with mass $M_{1}$ and charge $Q_{1}$ due to another body with mass $M_{2}$ and opposite charge $Q_{2}$ satisfying

$$
F_{12}=-2 \frac{e^{\prime 2}}{4 \pi} \frac{\left|Q_{1} Q_{2}\right|}{r^{2}},
$$

where $r=\left|x_{1}-x_{2}\right|$ is the distance between the two PeBHs. The factor of 2 comes from the fact that we have assumed these BHs are extremal and oppositely charged, so the force of gravity and of dQED are equal and add constructively. 
These charges form a time-varying dark electric dipole $\boldsymbol{p}=e^{\prime} Q_{1} \boldsymbol{x}_{1}+e^{\prime} Q_{2} \boldsymbol{x}_{2}$ that emits radiation as

$$
\frac{d E}{d t}=-\frac{2}{3} \frac{1}{4 \pi} \ddot{p}^{2}=-\frac{2}{3} \frac{\gamma^{2}}{4 \pi} \frac{1}{r^{4}},
$$

where $\gamma \equiv 2 e^{\prime 3}\left(\left|Q_{1}\right| / M_{1}+\left|Q_{2}\right| / M_{2}\right)\left|Q_{1} Q_{2}\right| /(4 \pi)$.

Using the standard expression for the separation between two orbiting bodies as a function of the semimajor axis $a$, eccentricity $e$, and angle that $\boldsymbol{r}$ makes with the periapsis $\theta$,

$$
r=\frac{a\left(1-e^{2}\right)}{1+e \cos \theta}
$$

as well as the eccentric anomaly to parametrize the motion as a function of time,

$$
\begin{aligned}
& t=\frac{T}{2 \pi}(\psi-e \sin \psi), \\
& (1-e \cos \psi)(1+e \cos \theta)=1-e^{2},
\end{aligned}
$$

the average energy loss over an orbital period $T$ is

$$
\begin{aligned}
\left\langle\frac{d E}{d t}\right\rangle & \approx-\frac{2}{3} \frac{\gamma^{2}}{4 \pi} \frac{1}{T} \int_{0}^{T} \frac{1}{r^{4}} d t \\
& =-\frac{2}{3} \frac{\gamma^{2}}{4 \pi} \frac{1}{a^{4}} \frac{1}{2 \pi} \int_{0}^{2 \pi}(1-e \cos \psi)^{-3} d \psi \\
& =-\frac{1}{3} \frac{\gamma^{2}}{4 \pi} \frac{1}{a^{4}} \frac{2+e^{2}}{\left(1-e^{2}\right)^{5 / 2}} .
\end{aligned}
$$

This can be related to the total energy

$$
E=-2 \frac{e^{\prime 2}\left|Q_{1} Q_{2}\right|}{(4 \pi)(2 a)} \equiv-\frac{\gamma^{\prime}}{2 a},
$$

by the expression

$$
\left\langle\frac{d E}{d t}\right\rangle \approx-\frac{16}{3} \frac{1}{4 \pi} \frac{\gamma^{2}}{\gamma^{\prime 4}} E^{4} \frac{2+e^{2}}{\left(1-e^{2}\right)^{5 / 2}} .
$$

Then, one can integrate $E$ from some initial $E\left(a_{i}\right)$ to $-\infty$ to calculate the merger time as

$$
\begin{aligned}
t & \approx \frac{1}{2} \frac{4 \pi \gamma^{\prime}}{\gamma^{2}} a^{3} \frac{\left(1-e^{2}\right)^{5 / 2}}{2+e^{2}} \\
& =\frac{4 \pi^{2} a^{3}}{e^{\prime 4}\left|Q_{1} Q_{2}\right|\left(\left|Q_{1}\right| / M_{1}+\left|Q_{2}\right| / M_{2}\right)^{2}} \frac{\left(1-e^{2}\right)^{5 / 2}}{2+e^{2}},
\end{aligned}
$$

where we have dropped the subscript $i$ in $a_{i}$. Finally, one can substitute $e^{\prime}|Q|=\sqrt{4 \pi} M / M_{\mathrm{pl}}$ to obtain (C3).

\section{APPENDIX E: ABSORPTION OF CHARGES}

To estimate how much charge is absorbed by the PBH after it has formed, starting from the time that the $\mathrm{BH}$ is formed with mass $M_{\mathrm{BH}}^{\text {init }}$, the nonrelativistic $\mathrm{PBH}$ will encounter and absorb relativistic dark electrons or positrons at a rate $n_{e^{\prime}} \sigma v$. Compared to the Hubble scale, the absorption rate is efficient as long as

$$
\frac{n_{e^{\prime}} \sigma v}{H} \approx 0.82 \frac{g}{\sqrt{g_{*}}}\left(\frac{T^{\prime}}{T}\right)^{3}\left(\frac{T M^{2}}{M_{\mathrm{pl}}^{3}}\right) \gtrsim 1,
$$

where $g$ is the number of degrees of freedom for $e^{\prime}$ and we have approximated $\sigma \approx \pi r_{+}^{2} \approx \pi\left(2 G_{N} M\right)^{2}$ when $M \gg$ $M_{\mathrm{eBH}}$ (when $M \approx M_{\mathrm{eBH}}, r_{+} \approx G_{N} M$ ). Here, we use the geometric cross section, which is valid when the wavelength of $e^{\prime}$ is shorter than the $\mathrm{BH}$ radius [1], i.e., $T^{\prime} \gtrsim$ $M_{\mathrm{pl}}^{2} /(2 M) \approx 10^{5} \mathrm{GeV} \times\left(10^{9} \mathrm{~g} / M\right)$ assuming $T^{\prime}>m_{e^{\prime}}$.

In the early stage before the PBHs have evaporated to near extremal, the gravitational interaction can be assumed under suitable model parameters to be stronger than the dark electromagnetic interaction, so one can approximately ignore the rate difference for dark electron and positron absorption. The net effects would be an increase of the variance $\sigma_{Q}$ and a slight change of the charge distribution. This absorption process may freeze out in a few different ways: (i) the number density of dark electrons and positrons decreases (e.g., after $T^{\prime}<m_{e^{\prime}}$ ), (ii) the PBH evaporates to a $\mathrm{PeBH}$ and its geometric cross section shrinks, or (iii) the dark electromagnetic force becomes important in repelling like-charged particles and attracting unlike-charged particles. Note that (iii) occurs automatically once the PBH evaporates to a PeBH if both $n_{e^{\prime}}$ and the geometric cross section are not too small and if the WGC holds. Since (iii) can lead to discharge, we will generally prefer that freeze-out occurs in a different way. This can be arranged, e.g., by having $T^{\prime}$ drop below $m_{e^{\prime}}$ and allowing the dark electrons or positrons to annihilate away before the $\mathrm{PBH}$ has time to evaporate, satisfying (i). Then, after the $\mathrm{BH}$ evaporates to (nearly) extremal, the charge neutralization rate can remain smaller than the Hubble rate, so the PeBH with a charge $Q$ can survive until the current universe.

Proceeding with these assumptions, it turns out that if the $\mathrm{PBH}$ formation temperature is much greater than the freezeout temperature, then most of the absorption occurs near the formation temperature. Still assuming that the PBH formed during radiation domination, the number of dark electrons and positrons absorbed can then be estimated by integrating $n_{e^{\prime}} \sigma v$, resulting in (10). 
[1] S. Hawking, Mon. Not. R. Astron. Soc. 152, 75 (1971).

[2] B. J. Carr and S. W. Hawking, Mon. Not. R. Astron. Soc. 168, 399 (1974).

[3] B. Carr, F. Kuhnel, and M. Sandstad, Phys. Rev. D 94, 083504 (2016).

[4] M. Sasaki, T. Suyama, T. Tanaka, and S. Yokoyama, Classical Quantum Gravity 35, 063001 (2018).

[5] B. J. Carr, K. Kohri, Y. Sendouda, and J. Yokoyama, Phys. Rev. D 81, 104019 (2010).

[6] H. Niikura et al., Nat. Astron. 3, 524 (2019).

[7] A. Katz, J. Kopp, S. Sibiryakov, and W. Xue, J. Cosmol. Astropart. Phys. 12 (2018) 005.

[8] Y. Bai and N. Orlofsky, Phys. Rev. D 99, 123019 (2019).

[9] S. W. Hawking, Commun. Math. Phys. 43, 199 (1975).

[10] Y. Aharonov, A. Casher, and S. Nussinov, Phys. Lett. B 191, 51 (1987).

[11] J. H. MacGibbon, Nature (London) 329, 308 (1987).

[12] S. W. Hawking, Phys. Rev. D 14, 2460 (1976).

[13] P. Chen, Y. C. Ong, and D.-H. Yeom, Phys. Rep. 603, 1 (2015).

[14] D. N. Page, Phys. Rev. D 14, 3260 (1976).

[15] J. S. Schwinger, Phys. Rev. 82, 664 (1951).

[16] G. W. Gibbons, Commun. Math. Phys. 44, 245 (1975).

[17] W. A. Hiscock and L. D. Weems, Phys. Rev. D 41, 1142 (1990).

[18] E. Sorkin and T. Piran, Phys. Rev. D 63, 124024 (2001).

[19] I. B. Khriplovich, Yad. Fiz. 65, 1292 (2002) [Phys. At. Nucl. 65, 1259 (2002)].

[20] I. B. Khriplovich, Phys. Rep. 320, 37 (1999).

[21] C.-M. Chen, J.-R. Sun, F.-Y. Tang, and P.-Y. Tsai, Classical Quantum Gravity 32, 195003 (2015).

[22] S. P. Kim, H. K. Lee, and Y. Yoon, Int. J. Mod. Phys. D 28, 1950139 (2019).

[23] N. Arkani-Hamed, L. Motl, A. Nicolis, and C. Vafa, J. High Energy Phys. 06 (2007) 060.

[24] J. Brown, W. Cottrell, G. Shiu, and P. Soler, J. High Energy Phys. 10 (2015) 023.

[25] E. D. Hall, R. X. Adhikari, V. V. Frolov, H. Müller, M. Pospelov, and R. X. Adhikari, Phys. Rev. D 98, 083019 (2018).

[26] A. Kawasaki, Phys. Rev. D 99, 023005 (2019).

[27] D. Carney, S. Ghosh, G. Krnjaic, and J. M. Taylor, arXiv: 1903.00492.

[28] K. Kohri and J. Yokoyama, Phys. Rev. D 61, 023501 (1999).

[29] M. Raidal, C. Spethmann, V. Vaskonen, and H. Veermae, J. Cosmol. Astropart. Phys. 02 (2019) 018.

[30] M. G. Aartsen et al. (IceCube Collaboration), Phys. Rev. D 98, 062003 (2018).

[31] P. W. Gorham et al. (ANITA Collaboration), Phys. Rev. D 99, 122001 (2019).

[32] O. Scholten, S. Buitink, J. Bacelar, R. Braun, A. G. de Bruyn, H. Falcke, K. Singh, B. Stappers, R. G. Strom, and R. al Yahyaoui, Phys. Rev. Lett. 103, 191301 (2009).

[33] S. Buitink, O. Scholten, J. Bacelar, R. Braun, A. G. de Bruyn, H. Falcke, K. Singh, B. Stappers, R. G. Strom, and R. A. Yahyaoui, Astron. Astrophys. 521, A47 (2010).

[34] M. G. Aartsen et al. (IceCube Collaboration), Adv. Space Res. 62, 2902 (2018).

[35] M. G. Aartsen et al. (IceCube Collaboration), Eur. Phys. J. C 78, 831 (2018).
[36] J. Pretz (HAWC Collaboration), Proc. Sci., ICRC2015 (2016) 820 .

[37] A. U. Abeysekara et al. (HAWC Collaboration), J. Cosmol. Astropart. Phys. 02 (2018) 049.

[38] B.S. Acharya et al. (CTA Consortium Collaboration), Science with the Cherenkov Telescope Array (World Scientific Publishing Co. Pte. Ltd., Singapore, 2018).

[39] M. Ackermann et al. (Fermi-LAT Collaboration), Astrophys. J. 761, 91 (2012).

[40] M. Ackermann et al. (Fermi-LAT Collaboration), Astrophys. J. 799, 86 (2015).

[41] E. Zas (Pierre Auger Collaboration), Proc. Sci., ICRC2017 (2018) 972.

[42] G. Tešić, Proc. Sci., ICRC2015 (2016) 328.

[43] H. A. Ayala Solares, Galaxies 7, 19 (2019).

[44] E. D. Stewart, Phys. Rev. D 56, 2019 (1997).

[45] S. M. Leach, I. J. Grivell, and A. R. Liddle, Phys. Rev. D 62 , 043516 (2000).

[46] M. Drees and E. Erfani, J. Cosmol. Astropart. Phys. 01 (2012) 035.

[47] M. Kawasaki, T. Takayama, M. Yamaguchi, and J. Yokoyama, Phys. Rev. D 74, 043525 (2006).

[48] T. Kawaguchi, M. Kawasaki, T. Takayama, M. Yamaguchi, and J. Yokoyama, Mon. Not. R. Astron. Soc. 388, 1426 (2008).

[49] P. H. Frampton, M. Kawasaki, F. Takahashi, and T. T. Yanagida, J. Cosmol. Astropart. Phys. 04 (2010) 023.

[50] S. Kasuya and M. Kawasaki, Phys. Rev. D 80, 023516 (2009).

[51] M. Kawasaki, N. Kitajima, and T. T. Yanagida, Phys. Rev. D 87, 063519 (2013).

[52] N. Orlofsky, A. Pierce, and J. D. Wells, Phys. Rev. D 95, 063518 (2017).

[53] B. J. Carr, Astrophys. J. 201, 1 (1975).

[54] Y. Akrami et al. (Planck Collaboration), Astrophys. Space Sci. 364, 69 (2019).

[55] N. Aghanim et al. (Planck Collaboration), arXiv:1807.06209.

[56] J. G. Skellam, JRSS 109, 296 (1946).

[57] J. Kang, M. A. Luty, and S. Nasri, J. High Energy Phys. 09 (2008) 086.

[58] C. Jacoby and S. Nussinov, arXiv:0712.2681.

[59] B. Holdom, Phys. Lett. 166B, 196 (1986).

[60] T. Gherghetta, J. Kersten, K. Olive, and M. Pospelov, Phys. Rev. D 100, 095001 (2019).

[61] J. Redondo, J. Cosmol. Astropart. Phys. 07 (2015) 024.

[62] D. N. Page, Phys. Rev. D 13, 198 (1976).

[63] D. N. Page, Phys. Rev. D 16, 2402 (1977).

[64] A. W. Graham, D. Merritt, B. Moore, J. Diemand, and B. Terzic, Astron. J. 132, 2701 (2006).

[65] G. Weidenspointner, M. Varendorff, S. C. Kappadath, K. Bennett, H. Bloemen, R. Diehl, W. Hermsen, G. G. Lichti, J. Ryan, and V. Schönfelder, AIP Conf. Proc. 510, 467 (2000).

[66] P. Sreekumar et al. (EGRET Collaboration), Astrophys. J. 494, 523 (1998).

[67] A. W. Strong, I. V. Moskalenko, and O. Reimer, Astrophys. J. 613, 956 (2004).

[68] M. Ahlers, Y. Bai, V. Barger, and R. Lu, Phys. Rev. D 93, 013009 (2016). 
[69] Y. Ali-Haımoud, E. D. Kovetz, and M. Kamionkowski, Phys. Rev. D 96, 123523 (2017).

[70] K. Ioka, T. Chiba, T. Tanaka, and T. Nakamura, Phys. Rev. D 58, 063003 (1998).
[71] M. Sasaki, T. Suyama, T. Tanaka, and S. Yokoyama, Phys. Rev. Lett. 117, 061101 (2016); 121, 059901(E) (2018).

[72] P. C. Peters, Phys. Rev. 136, B1224 (1964). 